\title{
A set of lateral shear optical tests with a liquid crystal display
}

\section{Conjunto de pruebas ópticas de desplazamiento lateral implementadas con una pantalla de cristal líquido}

\author{
J. Sánchez-Paredes ${ }^{(1)}$, G. Silva-Ortigoza ${ }^{(2)}$, J. Castro-Ramos ${ }^{(1, *}$, S. Vázquez-Montiel ${ }^{(3, A)}$ \\ 1. Instituto Nacional de Astrofísica Óptica y Electrónica, Ap. Postal 51 y 216, C.P. 72000, Tonantzintla, Puebla, México. \\ 2. Fac. Ciencias Físico Matemáticas, Benemérita Universidad Autónoma, Ap. Postal 1152, 72001, Puebla, México. \\ 3. Universidad Politécnica de Tulancingo, C.P. 43697,Tulancingo, Hidalgo, México. \\ ${ }^{(*)}$ Email: jcastro@inaoep.mx \\ A: miembro de AMO / AMO member \\ Received / Recibido: 12/02/2013. Revised / Revisado: 18/03/2013. Accepted / Aceptado: 03/04/2013. \\ DOI: http://dx.doi.org/10.7149/0PA.46.3.205
}

\begin{abstract}
:
In the literature it is possible to find the implementation of interferometric lateral shear tests with a liquid crystal display (LCD). We collected them and implemented others not previously reported. We show the versatility that the LCD offers to test of optical surfaces, so that graduate students can experience the following tests: the Foucault test, the Wire test, the Hartmann test, the Ronchi test and the null Ronchi test. Although these tests have been studied and developed since many years ago, they require different screens for each test. One advantage of using a LCD is that it can display many different screens, and therefore all mentioned tests can be developed. Another advantage is that, by digitizing the patterns observed with a CCD camera, it is possible to analyze them to obtain quantitative results instead of qualitative results; hence we use the Ronchi test as lateral shear interferometer to develop the phase shifting interferometry (PSI). In order to validate our method with the PC we implement the PSI using the Hariharan algorithm on the LCD. We used a LED as illuminating source avoiding the use of expensive sources of light. A Ronchi grating with a big period was required when testing optical surfaces with large $f / \#$, vice versa. This was done easily from the PC on the LCD, with a software that we developed with Visual C++ 6.0, a program to control the different parameters of the screens displayed on the LCD.
\end{abstract}

Key words: Null Ronchi Test, Foucault Test, Wire Test, Hartmann Test, Circular Grid Test, Phase Shifting, Liquid Crystal Display.

\section{RESUMEN:}

En la literatura se puede encontrar la implementación de pruebas interferométricas de desplazamiento lateral en una pantalla de cristal liquido (LCD). Las hemos recopilado y hemos implementado otras que no lo están. Mostramos la versatilidad que tiene la LCD en la prueba de superficies ópticas para que estudiantes de nivel superior puedan llevar a la práctica la prueba de Foucault, la prueba del alambre, la prueba de Hartmann, la prueba de Ronchi y la prueba de Ronchi nula. Aunque estas pruebas han sido estudiadas y desarrolladas desde hace muchos años, estas necesitan diferentes pantallas para que cada prueba sea implementada. Una ventaja de usar una LCD es que con ésta, es posible desplegar demasiadas pantallas en la LCD y por lo tanto se pueden desarrollar todas las pruebas mencionadas anteriormente. La otra ventaja es que digitalizando los patrones observados con una cámara CCD es posible analizarlos y obtener resultados cuantitativos en lugar de resultados cualitativos. También se utiliza la prueba de Ronchi como interferómetro de desplazamiento lateral para desarrollar la interferometría de desplazamiento de fase (PSI). Con una computadora personal (PC) implementamos la PSI con el algoritmo de Hariharan. Usamos un LED como fuente de iluminación evitando el uso de fuentes de iluminación costosas. Podemos probar cualquier sistema convergente de una manera cuantitativa. Al probar superficies ópticas con $f / \#$ grandes, necesitamos un período grande de la rejilla de Ronchi y viceversa, esto se logra desde una PC con software desarrollado en Visual C++ 6.0 el cual permite controlar los parámetros desplegados 
en la LCD para poder implementar las diferentes pruebas ópticas descritas.

Palabras clave: Prueba Nula de Ronchi, Prueba de Foucault, Prueba del Alambre, Pantalla de Cristal Líquido, Prueba de Rejilla Circular, Desplazamiento de Fase.

\section{REFERENCES AND LINKS / REFERENCIAS Y ENLACES}

[1]. J. Sánchez-Paredes, J. Castro-Ramos, S. Vázquez-Montiel, "A comparison between 2D phase unwrapping techniques", Proc. SPIE 6292, 62921K (2006).

[2]. D. Malacara, Edt., Optical Shop Testing, $3^{\text {rd }}$ Edt. John Wiley \& Sons Inc., New Jersey, USA (2007). DOI

[3]. V. Ronchi, "Forty years of history of a grating interferometer", Appl. Opt. 3, 437-451 (1964). DOI

[4]. J. M. Nuñez-Alfonso, A. Cordero-Davila, S. Vergara-Limon, J. Cuautle-Cortes, "Improved Ronchi tester", Appl. Opt. 40, 501-505 (2001).

[5]. M. Mora Gonzalez, N. Alcalá Ochoa, "The Ronchi test with LCD grating", Opt. Commun. 191, 203-207 (2001). DOI

[6]. J. Castro Ramos, S. Vázquez-Montiel, A. Padilla-Vivanco, "Phase shifting interferometry by using a LCD and bironchi test", Proc. SPIE 5662, 639-645 (2004). DOI

[7]. C.-W. Liang, C.-F. Ou, "Phase shifting grating-slit test utilizing a liquid crystal display", Opt. Rev. 16, 476-478 (2009). DOI

[8]. W. S. Meyers, H. P. Stahl, "Contouring of a free oil surface”, Proc. SPIE 1755, $84-94$ (1993). DOI

[9]. K. Omura, T. Yatagai, "Phase measuring Ronchi test", Appl. Opt. 27, 523-528 (1988). DOI

[10]. J. Sánchez-Paredes, G. Silva-Ortigoza, J. Castro-Ramos, J. Sasian, "1 $\lambda$ Ronchi tester to obtain the wave front aberration in converging optical systems using phase shifting interferometry", Opt. Pura Apl. 45, 461-473 (2012). DOI

[11].P. Hariharan, B. F. Oreb, T. Eiju, "Digital phase shifting interferometry: A simple error-compensating phase calculation algorithm", Appl. Opt. 26, 2504-2506 (1987). DOI

[12]. A. Cornejo-Rodriguez, "Ronchi test", Chapter 9 in D. Malacara, Edt., Optical Shop Testing, $3^{\text {rd }}$ Edt., J ohn Wiley \& Sons Inc., New Jersey, USA (2007).

[13]. Y. Cheng, J.C. Wyant, "Phase shifter calibration in phase shifting interferometry", Appl. Opt. 24, 30493052 (1985). DOI

[14]. R. Mukundan, K. R. Ramakrishnan, Moment Functions in Image Analysis: Theory and Aplications, pp. 937, pp. 127-128, World Scientific Publishing CO. Pte. Ltd., Singapore (1998).

[15]. T. Kreis, "Digital holographic interference-phase measurement using the Fourier-transform method", J. Opt. Soc. Am. A 3, 847-855 (1986). DOI

[16]. D. C. Ghiglia, L. A. Romero, "Robust two-dimensional weighted and unweighted phase unwrapping that uses fast transforms and iterative methods", J. Opt. Soc. Am. A 11, 107-117 (1994). DOI

[17]. Durango software is a trademark of diffraction international ${ }^{\circledR}$ in: http://www.diffraction.com/ (Last visited: Sep., 10 ${ }^{\text {th }}$ 2013).

[18]. G. W. Ritchey, On the Modern Reflecting Telescope and the Making and Testing of Optical Mirrors", Smithsonian Contributions to Knowledge Vol XXXIV (1904)

[19]. J. Castro-Ramos, Jose Sasian, "Automatic phase shifting Ronchi tester with a square Ronchi ruling", Proc. SPIE 5532, 199-210 (2004). DOI

[20]. H. Schreiber, J. H. Bruning, "Phase shifting interferometry", Chapter 14 in D. Malacara, Edt., Optical Shop Testing, $3^{\text {rd }}$ Edt., John Wiley \& Sons Inc., New Jersey, USA (2007)..

[21]. K. Creath, "Phase measurement interferometry techniques", Prog. Optics 26, 349-393 (1998). DOI

[22]. A. Cornejo, D. Malacara, "Ronchi test of aspherical surfaces, analysis and accuracy", Appl. Opt. 9, 19871901 (1970).

[23]. A. Cordero-Dávila, E. Luna-Aguilar, S. Vázquez-Montiel, S. Zarate-Vázquez, M. E. Percino-Zacarias "Ronchi test with a square grid", Appl. Opt. 37, 672-675 (1998). DOI

[24]. M. V. R. K. Murty, A. H. Shoemaker, "Theory of concentric circular grid", Appl. Opt. 5, 323-326 (1965). DOI 
[25]. A. Morales, D. Malacara, "Geometrical parameters in the Hartmann test of aspherical mirrors", Appl. Opt. 22, 3957-3959 (1983). DOI

[26].P. Su, R. Parks, L. Wang, J. Burge, R. Angel, "A new test for optical surfaces", SPIE Newsroom 20 January (2011).

[27]. D. Malacara, A. Cornejo, "Null Ronchi test for aspherical surfaces", Appl. Opt. 13, 1778-1780 (1974). DOI

[28]. C. Guo, X. Su, W. Chen, B. Lei, F. Wu, "Three-dimensional shape measurement of large-aperture aspheric mirrors by off-axis null Ronchi test", Appl. Opt. 51, 1276-1282 (2012). DOI

[29].K. E. Atkinson, An Introduction to Numerical Analysis, $2^{\text {nd }}$ Edt., John Wiley \& Sons, New York, USA (1989).

\section{Introduction}

Since Vasco Ronchi developed the Ronchi test [1$3]$, it has been studied and improved by several authors for a long time. Nuñez and Cordero [4] proposed to use a LED as a source of light in the Ronchi test. The LCD has been used as Ronchi grating in the Ronchi test using a laser as source of illumination [5-8] and it has been developed by different authors but they obtain poor resolution and visibility. Omura \& Yatagai [9], Sanchez and Silva [10] published a Method to test lenses, which uses a step motor with a PC interface to move a Ronchi ruling and applying the Hariharan [11] algorithm the wrapped wavefront phase was calculated. Using a conventional polished LED as illumination source we avoid the use of expensive lasers and also the use of tungsten lamp as source of illumination in the Ronchi test. With this configuration we did not use additional optics to observe the common Ronchi patterns as Cornejo has shown in their experimental setup [12]. In this paper we propose to implement a set of lateral shear optical tests which still do not have been implemented on a liquid crystal display (LCD), in spite of many years have passed these tests still are very used when somebody try to test large astronomical telescopes, microscope objectives and other optical instruments. Using a LCD as screen we avoid to buy an expensive set of Ronchi rulings, also we avoid employing so much time building Hartmann screens and others. Furthermore to elaborate null Ronchi gratings always has been a problem; with the software developed this is done almost instantaneously. Additionally we not only give qualitative results we even show that with the software developed is possible to obtain quantitative results. By showing the implementation of the Hartmann test and the bironchi test we avoid to use expensive precision mounts to rotate Ronchi rulings, also is reported the phase shifting technique without using stepping motors or a piezoelectric or another device, avoiding the phase shift errors as Cheng and Wyant compensated [13]. The problem starts in next section where the basic theories about the implemented lateral shear optical tests are shown. In section three is described the PSI technique and the Hariharan algorithm, also is explained a method to filter the valid pixels of the fringe patterns from the invalid pixels based in geometrical moments which were described by Sanchez [10] and Mukundan [14]; they used it as a powerful tool to obtain a mask of the valid phase, and is described the algorithm used to unwrap the wavefront phase. Kreis [15] and Ghiglia [16] methods were used to unwrap the wavefront aberration phase and is described the trapezoidal rule used to integrate data. In section four is proposed an experimental setup for simultaneously to work with the knife edge test, the wire test, the Ronchi test, the bironchi test, the null Ronchi test and the Hartmann test on the LCD. We elaborate a $\mathrm{C}++$ program to be handled for any graduate student whom whish apply these interferometric tests. Also it is explained the resolution of the gratings displayed on the LCD and finally our experimental results were compared with Durango software $^{\mathrm{TM}}$ [17] to show the validity of our implemented tests on the LCD; finally conclusions are given. 


\section{Optical tests}

\subsection{Ronchi test}

We use the geometrical model of the Ronchi test [3] for the fringe formation that explains the fringes as projections of the Ronchi grating by the rays that fall on it. The Ronchi test measures the transverse aberration, which is directly related to the derivative of the wavefront. Let $r$ be the distance to the vertex of the surface test to the Ronchi grating, $d$ the period of the grating, and $\varphi$ the angle that the ruling makes with the $y$ axis. Then:

$\frac{\partial W(x, y)}{\partial x} \cos \varphi-\frac{\partial W(x, y)}{\partial y} \sin \varphi=-\frac{m(x, y) d}{R}$

where $W(x, y)$ is the wavefront aberration function respect to its osculating sphere, $m(x, y)$ is the fringe order function.

Equation (1) gives us the relation between fringe order and the wavefront aberration derivative, the intensity of the fringe pattern is related to the fringe order. This may be found by back projecting the Ronchi grating to the mirror according to the next equation:

$$
I(x, y)=A(x, y)+B(x, y) \cos [\theta(x, y)+\delta],
$$

where

$$
\begin{aligned}
& \theta(x, y)= \\
& =\frac{2 \pi}{d} R\left[\frac{\partial W(x, y)}{\partial x} \cos \varphi-\frac{\partial W(x, y)}{\partial y} \sin \varphi\right]
\end{aligned}
$$

is the phase of the intensity of the Ronchi pattern, $\delta$ is the initial phase of the grating and $A(x, y), B(x, y)$ are the background and contrast functions respectively.

\subsection{Foucault test}

The knife-edge method, introduced by Foucault has proved to be extremely useful for testing optical surfaces. Rays may be moved from their expected trajectories by optical aberrations, by diffraction or by a deformed, unfinished surface. The basic idea is to detect lateral displacements of rays by blocking out or modifying these displaced rays. The blocking or modification can be accomplished by placing screens in any of the planes of convergence of the light passing through, or being reflected from the optical

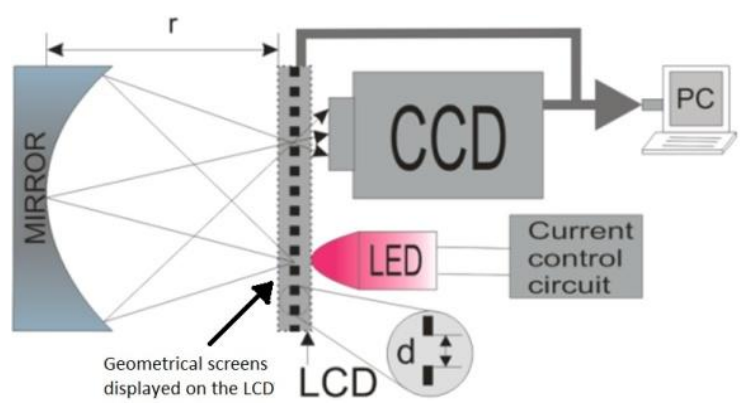

Fig. 1. Diagram of the Ronchi test with the LCD and LED.

surface under test. The main advantages are their high sensitivity and their simplicity both in apparatus and in qualitative interpretation. In general, this test is used to detect the presence of transversal aberrations; this is done displaying awide black line on the LCD of Fig. 1, simulating the knife edge that is blocking out part of a plane transverse by rays or diffracted light so that a shadow appears over the aberrated region.

\subsection{The wire test}

Foucault test is very sensitive for detecting zonal errors; however the Foucault test is less sensitive in measuring the errors. Ritchey [18] suggested using a screen with zonal slots over any aspherical surface, when testing mirrors by the Foucault method. In this way it is possible to calculate the amount of transversal ray aberration of the two slots of the zone, which are localized at opposite sides of the optical axis, to check whether the regions behind the slots are regular and to match between them. Those slots are displayed on the LCD of Fig. 1.

\subsection{Bironchi test and circular grid}

When a square grid $[19,23]$ is displayed on the LCD in the configuration of Fig. 1 the grid allow to process the transversal aberration of both the $\mathrm{X}$ and $\mathrm{Y}$ directions with only one series of ronchigrams. Also instead of straight lines or square grids, it is possible to use circular curves [24]. Patterns with this kind of ruling differ from the normal Ronchi patterns.

\subsection{Hartmann test}

Hartmann test $[25,26]$ is a screen test for quality examination of an optical system. This test needs a screen, Hartmann plate, which have a number of holes in it at the exit pupil of the optical 
system under test. We locate the LCD just in front of the optical system. The spots corresponding to these holes are recorded on a CCD, before or after focus as in Fig. 1. The positions of these spots are mapped back onto the optical element, and the difference between the positions of these spots and the holes of the screen are used to compute the aberrations.

\subsection{Null Ronchi test}

Also null Ronchi gratings $[27,28]$ can be displayed on the LCD for quality examination of optical systems. This test needs a null Ronchi screen that must be calculated and fabricated before that the test is realized. With the LCD we display on it the null Ronchi screen calculated avoiding the fabrication of the screen.

All the tests mentioned above can be implemented just with the configuration in Fig. 1 , by obtaining qualitative results.

\section{Basic theory to obtain quantitative results}

Qualitative optical test are not sufficient to characterize an optical surface, we need to obtain quantitative results with the configuration of the Fig. 1. We implement the PSI displaying horizontal and vertical Ronchi rulings on the LCD, with a CCD camera we digitize the fringe patterns observed and with Hariharan algorithm we recover the wrapped phase of the system under test. The wrapped phase needs to be unwrapped, to do we use the Kreis and Ghiglia methods. Experimentally the digitized patterns has two regions, one is with valid phase pixels that correspond to the exit pupil of the surface under test and the other is with nonvalid phase pixels that corresponds to the background, if we desire to obtain good quantitative results we must to differentiate valid phase pixels from non-valid phase pixels before doing the unwrapping process, to do that selection we use geometrical moments. The unwrapped phase obtained is the wavefront derivate and we need to integrate it to recover the wavefront, this is done with the trapezoidal rule.

\subsection{PSI and Hariharan algorithm}

The PSI [6,7] is not a hardware optical configuration. It is rather a way to get data and an analysis method that can be applied to a great variety of tests. Equation (2) is the fundamental equation of PSI. The intensity at each point varies as a sinusoidal function of the introduced phase shift $\delta$ with a temporal offset given by the unknown wavefront phase.

The Hariharan algorithm [11] is the PSI algorithm that we use to get the wrapped phase of the measured transversal aberration. It is insensitive to reference phase shift calibration errors. It uses five measurements of the Ronchigram intensity, and initially assumes a linear phase shift of $\alpha=90^{\circ}$ between frames:

$$
\delta_{i}=-2 \alpha,-\alpha, 0,+\alpha,+2 \alpha ; \quad i=1,2,3,4,5 .
$$

Equation (5) denotes the Hariharan algorithm. It takes five fringe patterns with a $90^{\circ}$ phase shift step:

$$
\phi(x, y)=\tan ^{-1}\left[\frac{2\left(I_{2}(x, y)-I_{4}(x, y)\right)}{2 I_{3}(x, y)-I_{5}\left((x, y)-I_{1}(x, y)\right.}\right] .
$$

\subsection{Geometrical moments}

While we have the five Ronchigrams, we made a intensities comparison between pixel to pixel for all Ronchigrams. Then, if the comparison does not change, we put a value 0 to that pixel in a mask that correspond for non-valid phase pixel and vice versa for valid phase pixels. Then geometrical moments [14] are applied to the mask to find the radius of the ellipse and the orientation angle of the valid phase pixels mask.

The geometrical moments $[10,14]$ were implemented to found the radius of the ellipse and the orientation angle of the simulated and experimental interferometric frames to compute the valid phase pixels mask. The major $a$, and minor $b$ radius, and the inclination angle $\theta$ of the ellipse are given by:

$$
\begin{aligned}
& a=2 \sqrt{\frac{I_{1}}{\mu_{00}}}, \\
& b=2 \sqrt{\frac{I_{2}}{\mu_{00}}},
\end{aligned}
$$




$$
\theta=\frac{1}{2} \tan ^{-1}\left(\frac{2 \mu_{11}}{\mu_{20}-\mu_{02}}\right)
$$

where

$$
\begin{gathered}
\mu_{p q}=\iint_{\tau}\left(x-x_{0}\right)^{p}\left(y-y_{0}\right)^{q} d x d y, \\
I_{1}=\left(\mu_{20}+\mu_{02}\right)+\frac{1}{2} \sqrt{\left(\mu_{20}+\mu_{02}\right)^{2}+4 \mu_{11}^{2}}, \\
I_{2}=\left(\mu_{20}+\mu_{02}\right)-\frac{1}{2} \sqrt{\left(\mu_{20}-\mu_{02}\right)^{2}+4 \mu_{11}^{2}} .
\end{gathered}
$$

\subsection{Phase unwrapping}

When phase shifting interferometry is used, we must correct the discontinuities produced by the arc tangent function (Eq. (5)), which is only defined from $-\pi / 2$ to $+\pi / 2$. The first correction is to extend the range from 0 to $2 \pi$. We use the Tomas Kreis [15] method that consist on calculate the wrapped phase differences between two pixels. If the value of the phase difference is less than $\pi$, the value of the unwrapped phase $\phi_{i, j}$ remains equal. If the value of the phase difference is bigger or equal than $\pi$, we have to subtract $2 \pi$ to the phase value, and if the value of the phase difference is fewer or equal than $-\pi$ we have to add $2 \pi$ to the phase value. The Ghiglia method [16] is the other method that we use to unwrap the phase. It consists basically in taking the differences of the wrapped phase with their adjacent neighbors with Eq. (8), and calculate the phase modulus $2 \pi$.

The phase difference $x_{i, j}$ is given by:

$$
x_{i, j}=-4 \varphi_{i, j}+\varphi_{i-1, j}+\varphi_{i, j-1}+\varphi_{i+1, j}+\varphi_{i, j+1},
$$

where $\varphi_{i, j}$ are the valid phase wrapped pixels. We then calculate the Bidimensional Discrete Fourier Cosine Transform (BDFCT). And then we calculate the following filter:

$$
\hat{\phi}_{i, j}=\frac{\hat{\rho}_{i, j}}{2\left(\cos \left(\frac{\pi i}{M}\right)+\cos \left(\frac{\pi j}{N}\right)-2\right)},
$$

where $\hat{\rho}_{i, j}$ are the values of the (BDFCT). Finally, the inverse bidimensional discrete Fourier cosine transform is used to obtain the unwrapped phase.

\subsection{Trapezoidal rule}

When we analyze Ronchigrams or Hartmanngrams, we are recovering the wavefront derivate. That is why, when the valid phase is correctly unwrapped, it is necessary to integrate it. We use the Simpson rule to recover the wavefront aberration since it is a technique useful for approximating the definite integral, and gives better approximations than trapezoidal rule [29].

\section{Experimental setup and software}

The experimental setup to implement the knife edge test, the wire test, the Ronchi test, the bironchi test, the null Ronchi test and the Hartmann test on the LCD is shown in Fig. 3. This arrangement consists of the mirror or lens under test, a LCD, which allows changing instantaneously different screens and automatically the pitch of the Ronchi ruling, and an interchangeable revolver of LEDs, which acts as source of illumination, none of them using any additional optical components, thus avoiding possible additional aberrations during the test. The LEDs were cut and polished until the wire bond to increase their numerical aperture and lighting angle. We use several LEDs, which cover the wavelength range 450-950 $\mathrm{nm}$, which increase the visibility of polished or roughness surfaces. We feed all LEDs employing a small power supply and a variable resistor to vary voltages, in order to have the correct current and voltage to increase or reduce the intensity of the LED in use. Ronchigrams were detected with a monochrome CCD camera of $640 \times 480$ pixels and, finally, a software program to display the screens on the LCD was made in Visual C++, as

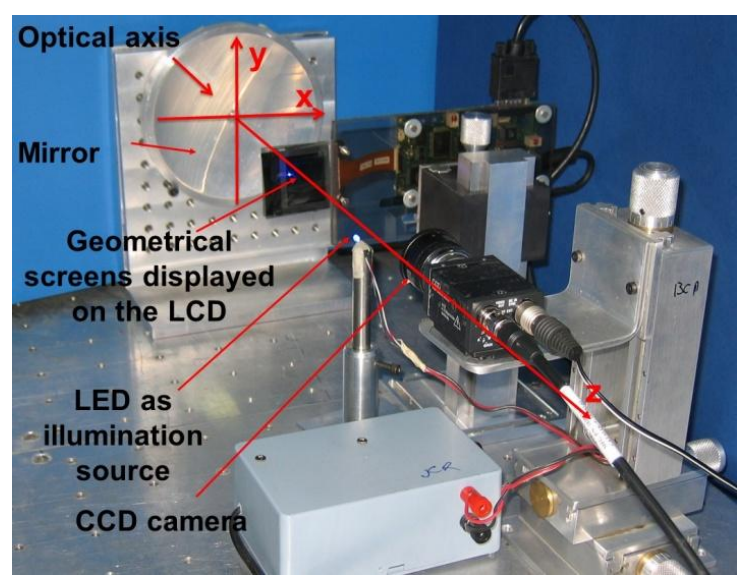

Fig. 3. Experimental setup used to implement lateral shear optical tests. 
well as other programs to compute transversal aberration and to compute the wavefront aberrations in an automatic process of the developed method.

\subsection{Resolution of the liquid crystal displayed grating with phase shift}

We use a LCD with $1024 \times 768$ pixels, and assume an ideal display with pixel size of $13 \mu \mathrm{m} \times 15 \mu \mathrm{m}$. In order to implement the phase shifting interferometry using the LCD to display Ronchigrams, it is necessary to avoid any diffraction phenomena $[5,12,21]$. Therefore, we need to calculate the period of the Ronchi rulings. The following Eq. (10) was used to calculate the minimal period on pixels per fringe:

$$
P F=\frac{360}{d f r},
$$

where $P F$ is the number of pixels per fringe (this quotient should be an integer) and $d f r$ is the phase step between Ronchi rulings; hence the range of the phase shift to make PSI goes from $1^{\circ}$ to $180^{\circ}$.

The LCD avoids the use of many expensive Ronchi rulings and screens, thus the optical test becoming more versatile because we can generate screens with different pitch. We use the LCD as a wire (one row of pixels), as a knife edge (one column of pixels), as a Ronchi grating (either several rows or columns of pixels), as a bironchi grating (both several rows and columns of pixels), and as a circular grating (radial test and to align optical surfaces). Even the null Ronchi test was implemented, with this experimental setup. Displaying horizontal and vertical Ronchi rulings the phase was shifted just by changing the pixel position of the grating displayed on the LCD and also we change the period of the displayed grating depending on the surface with small or big focal ratio. All of this was done easily with the developed software.

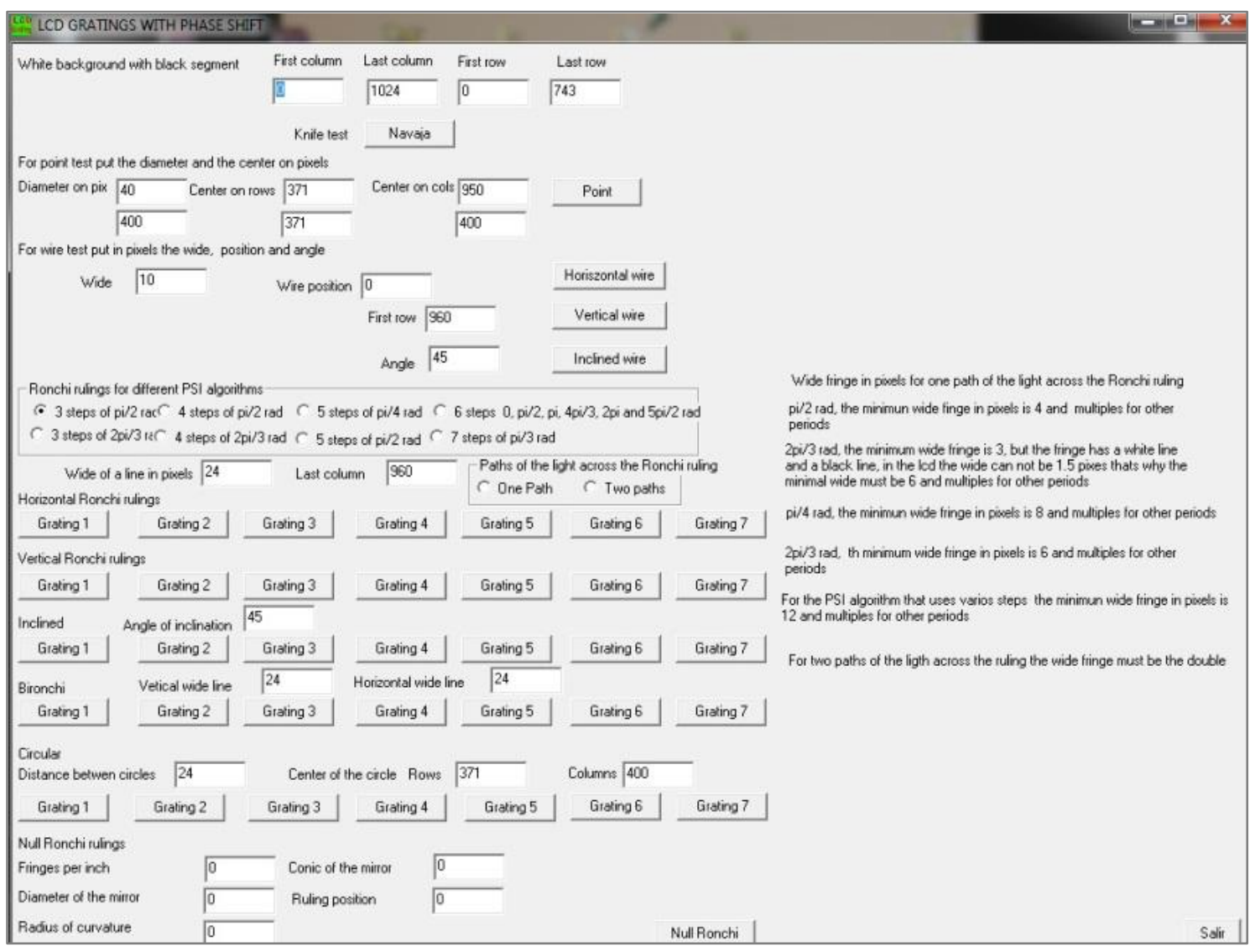

Fig. 4. Main window of the software used to display different screens on the LCD, and to analyze the digitized fringe patterns. 


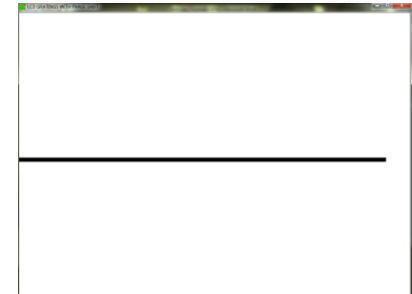

(a)

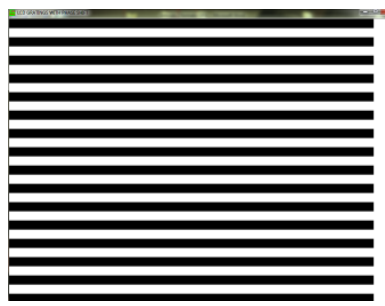

(d)

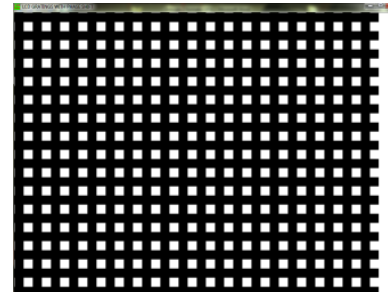

(g)

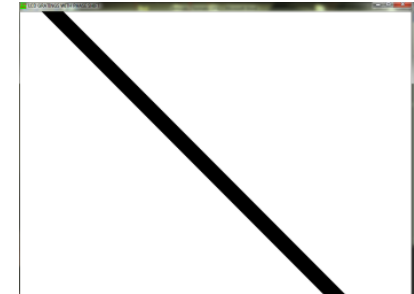

(b)

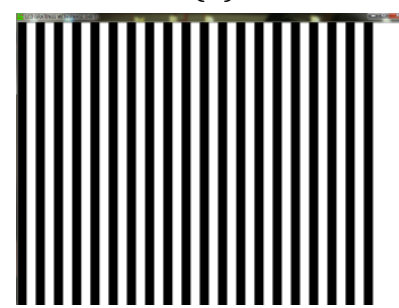

(e)

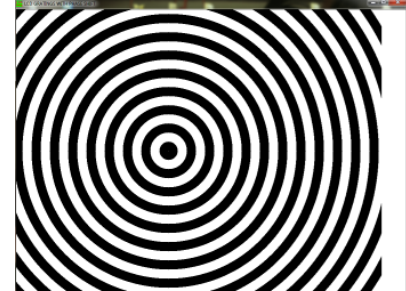

(h)

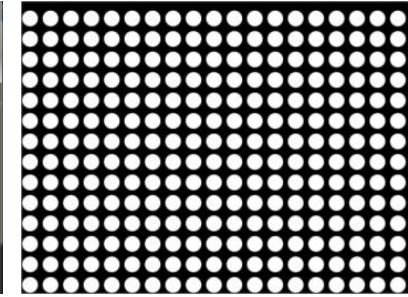

(c)

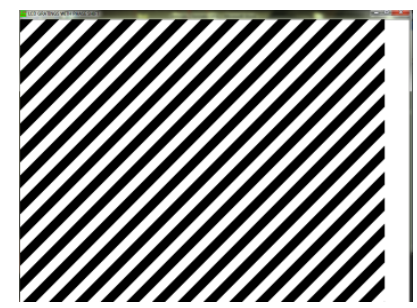

(f)

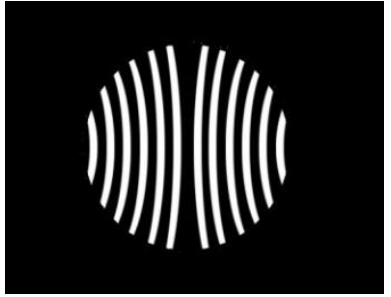

(i)

Fig. 5. Different kind of screens displayed on the LCD: a) horizontal wire,b)inclined wire,c) Hartmann screen, d) horizontal Ronchi ruling, e) vertical Ronchi ruling, f) inclined Ronchi ruling, g) bironchi ruling, h) circular ruling and i) null Ronchi ruling.

\subsection{Developed software}

In Fig. 4 we show the main window of the software that we developed. With it one can directly select the stuff that is displayed on the LCD: the kind of test, the pitch of the screen and the phase step. Also we can display almost any kind of screens with pitch between 2-512 pixels, to test any converging optical system with different $f / \#$. With this software we can recover the wrapped phase and select the method that someone prefer to unwrap the phase. This software computes the wavefront and the transversal aberration. Finally Zernike polynomials are provided for fitting the wavefront aberration of the optical system under test [10].

\subsection{Screens displayed on the LCD}

Next we show the implemented screens optical tests on the LCD that were applied with the method developed. In Figs. 5(a) and 5(b), we show that a wire can be displayed on the LCD and we can change the width of the black band. It can be placed at any place into the area of the
CCD and with this we can do even the knife test making the wire wider and placing it where is necessary. In Fig. 5(c) we show a Hartmann plate that can be displayed on the LCD. In Figs. 5(d), 5(e) and 5(f) we show that a Ronchi ruling can be displayed on the LCD with any orientation angle and we can change the period of the fringes from the program, avoiding the use of many different Ronchi rulings. In Fig. 5(g) we show that even a bironchi grating can be displayed on the LCD, and also how we can change the period of the fringes. And in Fig. 5(h) we show that even a circular grating can be displayed on the LCD. All these gratings can be shifted on the LCD obtain fringe patterns of the surface under test with the phase shifting technique, allowing us to avoid the use of stepping motors or piezo electric devices usually employed to perform such phase shift. And in Fig. 5(i) we show that even null Ronchi gratings can be displayed on the LCD. 


\section{Results}

Figure 6 shows some fringe patterns obtained with the experimental setup of Fig. 3, using gratings as shown in Fig. 5. The period of the Ronchi rulings displayed on the LCD was 24 pixels per fringe, which is equivalent to 30.2 fringes per inch. In Fig. 6(a) a wire pattern is obtained by displaying a white line as screen on the LCD. In Fig. 6(b), a knife pattern is obtained displaying black line on the LED. In Fig. 6(c), vertical fringe patterns were digitized when we display a vertical Ronchi ruling on the LCD. Figure 6(d) shows a horizontal fringe pattern digitized when we display a horizontal Ronchi ruling on the LCD. Figure 6(e) shows a tilted fringe pattern digitized when we display a tilted Ronchi ruling on the LCD. Figure 6(f) shows a fringe pattern digitized when we display a

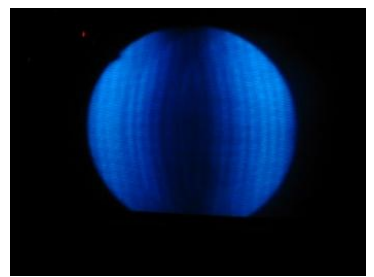

(a)

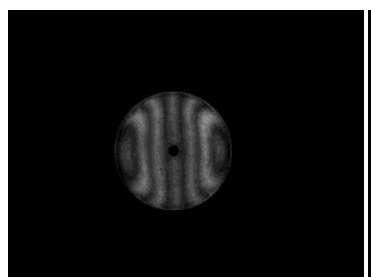

(c)

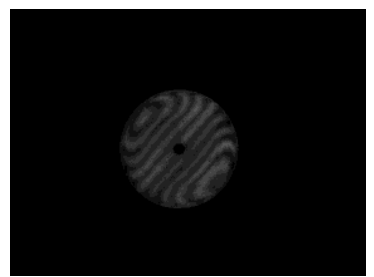

(e)

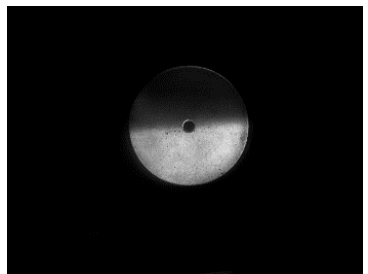

(b)

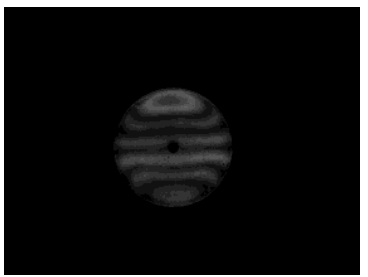

(d)

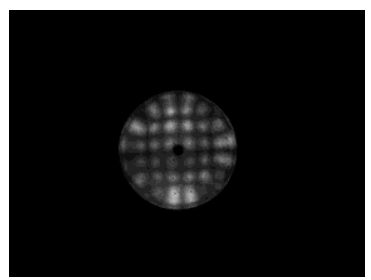

(f)

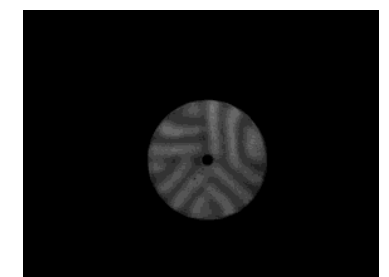

(g)

Fig. 6. Fringe patterns obtained experimentally for: (a) wire pattern, (b) knife pattern, (c) vertical fringe pattern, (d) horizontal fringe pattern, (e) tilted fringe pattern, (f) bironchi grating, and (g) multiple fringe patterns.

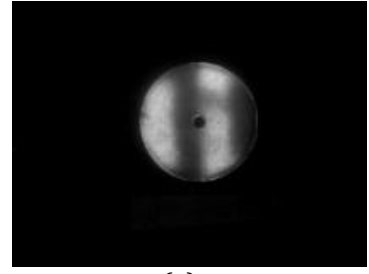

(a)

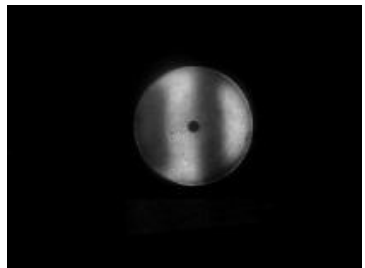

(c)

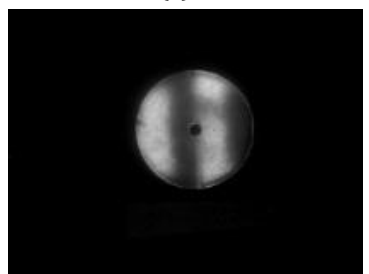

(e)

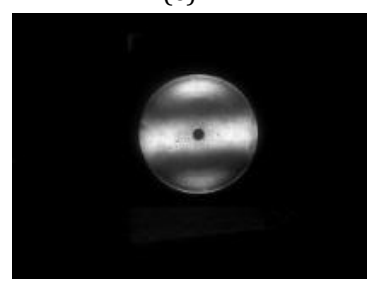

(g)

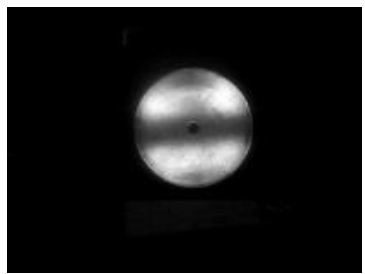

(i)

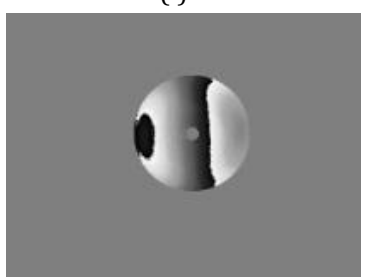

(k)

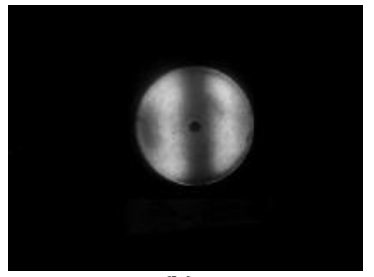

(b)

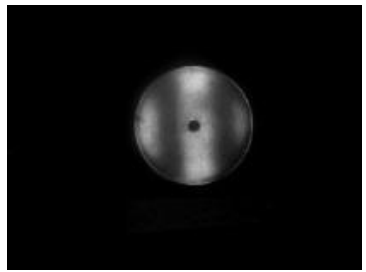

(d)

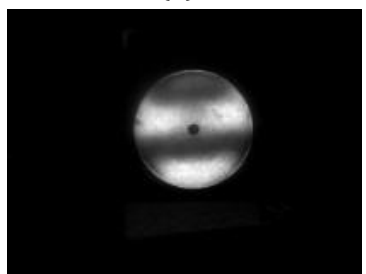

(f)

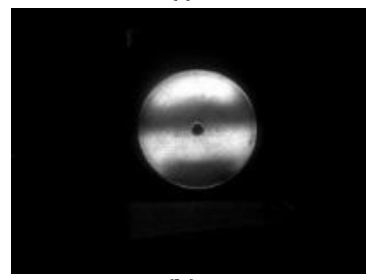

(h)

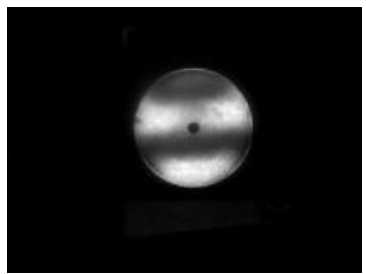

(j)

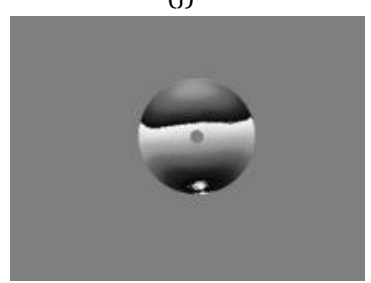

(1)

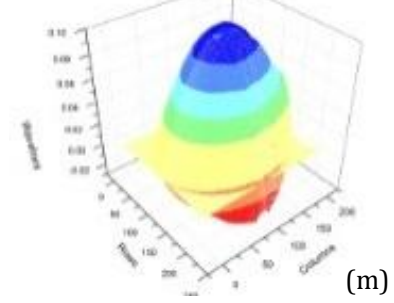

Fig. 7. Fringe patterns obtained experimentally with some intensity background. (a)-(e) show fringe patterns with horizontal phase shift. (f)-(j) show fringe patterns with vertical phase shift. (k) shows horizontal phase transversal aberration. (l) shows vertical phase transversal aberration and $(\mathrm{m})$ shows the wavefront aberration. 
bironchi ruling $[19,23]$ on the LCD. And, finally, Fig. 6(g) shows a multiple fringe pattern digitized when we display a multiple ruling on the LCD, which was generated specifically to show how versatile can be the test using the LCD as screens for the experimental setup (for example null Ronchi test) [25]. In all figures we see the fringe patterns digitized which shows a good contrast although we test mirrors with high roughness. This is due to the fact that we can vary instantaneously the wavelength, the pitch and the intensity of the LED used as source of illumination.

In order to show quantitative results PSI and Hariharan algorithm were implemented with Ronchi and Hartmann tests. Figure 7 shows five horizontal Ronchigrams (a-e), five vertical Ronchigrams (f-j), the vertical (k) and horizontal (l) transversal aberration and, finally, the reflected wavefront (m) coming from an 6065 aluminum mirror used as surface under test. This mirror has a diameter of $400 \mathrm{~mm}$, a radius of curvature of $150 \mathrm{~mm}$, and a hole in the center.

We can see that, when we analyze fringe patterns obtained at the workshop, it is very important to make a good valid phase pixels detection for the correct analysis. To verify our software we made a comparison versus Durango software ${ }^{\circledR}$ [17]. We tested a hyperbolic glass mirror in a Zygo interferometer and analyzed it with the Durango software, that uses the
Hariharan algorithm to make the interferograms analysis. The results show good agreement.

\section{Conclusion}

An experimental set up of lateral shear optical tests was implemented using a LCD to display several screens. We show that a LED as source illumination can be used without having problems with the visibility that is present in interferograms obtained with a laser or tungsten lamps. We have the advantage to generate and display different kinds of gratings with different pitch, making the tests more versatile. With the LCD, it was possible to implement the Foucault test, the wire test, the Hartmann test, the Ronchi test, the bironchi test and the null Ronchi test. By analyzing the Ronchigrams with PSI we obtained quantitative results. All the screens displayed on the LCD were generated with a software that we developed on visual $\mathrm{C}++6.0$.

\section{Acknowledgements}

This work was financially supported by CONACyT, Mexico, under project J50614. GSO thanks to SNI. Also thanks to F. SalomonGranados, E. Percino-Zacarias, A. Cordero-Davila, G. Gordiano-Alvarado, L. Sastre-Juarez, and the reviewers for your help to improve this paper. 